\title{
MAXIMUM ALPHA TO MINIMUM FISSION PULSE AMPLITUDE FOR A PARALLEL- PLATE AND HEMISPHERICAL CF-252 ION- CHAMBER INSTRUMENTED NEUTRON SOURCE
}

\author{
R. B. Oberer
}

September 2000 
ORNL/TM-2000/290

\section{MAXIMUM ALPHA TO MINIMUM FISSION PULSE AMPLITUDE FOR A PARALLEL-PLATE AND HEMISPHERICAL CF-252 ION-CHAMBER INSTRUMENTED NEUTRON SOURCE}

Richard B. Oberer

September 2000

Prepared by

OAK RIDGE NATIONAL LABORATORY

Oak Ridge, Tennessee 37831-6004

managed by

UT-BATTELLE, LLC

for the

U.S. DEPARTMENT OF ENERGY

under contract DE-AC05-00OR22725 


\section{CONTENTS}

Page

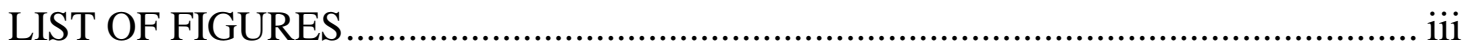

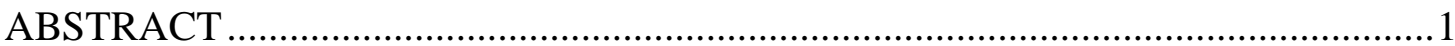

1. INTRODUCTION

2. FISSION FRAGMENT STOPPING POWER .....................................................2

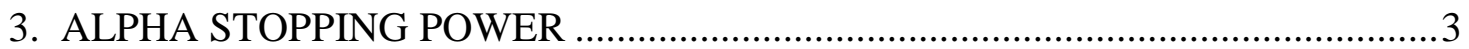

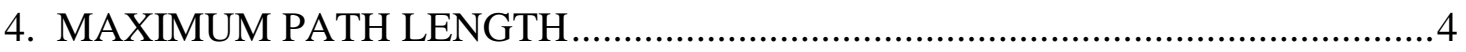

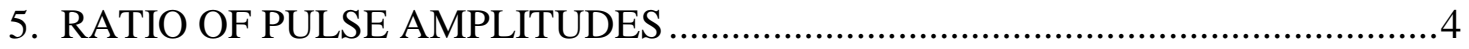

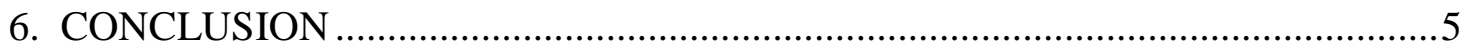

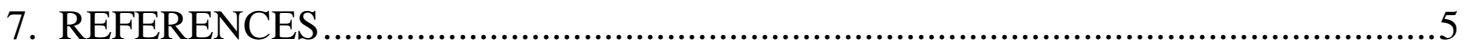




\section{LIST OF FIGURES}

Page

Fig. 1 Illustration of the a) parallel-plate and b) hemispherical configuration of a Cf-252 ion-chamber instrumented neutron source along with definition

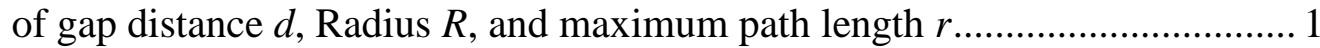

Fig. 2 The energy of an alpha particle as it traverses argon at $1 \mathrm{~atm}$ at room temperature. The stopping power, which is the slope of this curve is also shown.

Fig. 3 Percent minimum fission pulse to maximum alpha pulse in a parallel-plate and hemispherical fission chamber with a gap of $1 \mathrm{~mm}$ and varying $R$. Data for the $12.5 \mathrm{~mm}$ radius are shown as a square and circle, respectively. ........... 4 


\begin{abstract}
In an instrumented Cf-252 neutron source, it is desirable to distinguish fission events which produce neutrons from alpha decay events. A comparison of the maximum amplitude of a pulse from an alpha decay with the minimum amplitude of a fission pulse shows that the hemispherical configuration of the ion chamber is superior to the parallelplate ion chamber.
\end{abstract}

\title{
1. INTRODUCTION
}

This report compares the maximum alpha pulse contribution in two configurations of a Cf-252 ion-chamber instrumented neutron source. The two configurations are shown in Figure 1. The first configuration is a parallel-plate ion chamber. The second configuration is comprised of two concentric hemispheres. In both cases, Cf-252 is plated on a $1 \mathrm{~cm}$ diameter spot. In the parallel plate configuration the spot is centered on one plate. In the concentric-hemisphere configuration the spot is located on the smaller hemisphere.

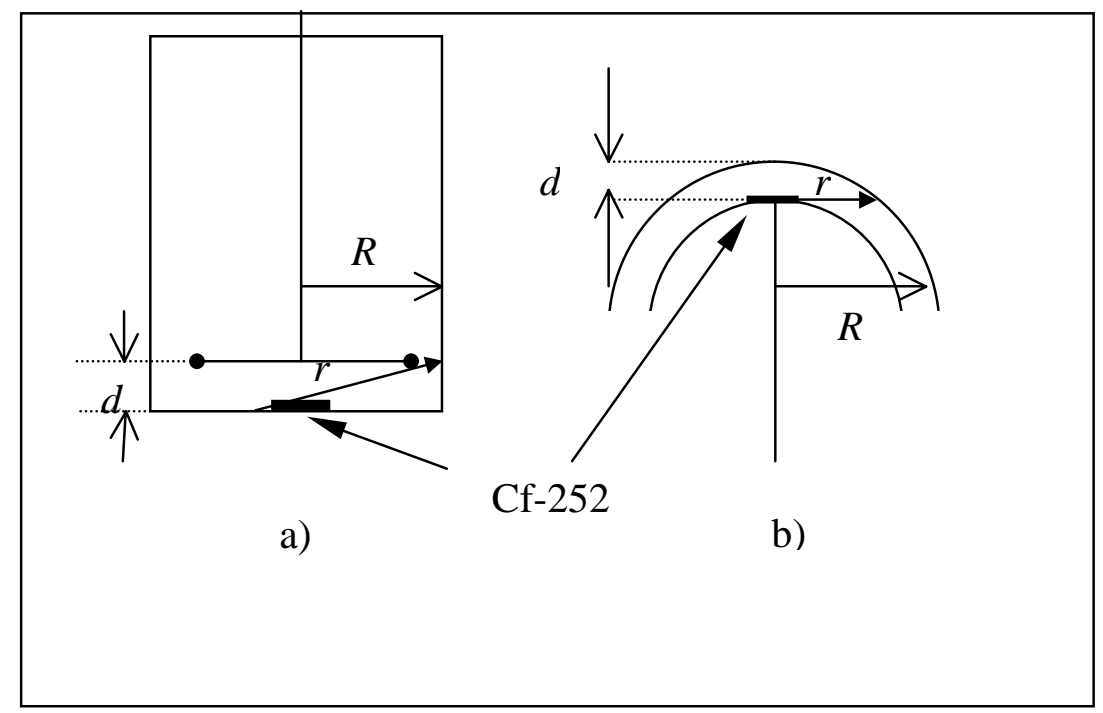

Figure 1. Illustration of the a) parallel-plate and b) hemispherical configuration of a Cf-252 ion-chamber instrumented neutron source along with definition of gap distance $d$, Radius $R$, and maximum path length $r$.

In an instrumented neutron source, it is desirable to distinguish fission events which produce neutrons from alpha decay events. A comparison of the maximum amplitude of a pulse from an alpha decay with the minimum amplitude of a fission pulse is therefore relevant. Both parallel-plate and hemispherical chambers have been built at ORNL (Refs. 1, 2, and 3). Unfortunately, none of the hemispherical sources are now operational. In one study the ratio of the maximum alpha pulse to the minimum fission fragment was reported to be $8 \%$ in the hemispherical configuration, approximately a factor of three better than the parallel plate configuration (Ref. 2). These results are in 
agreement with the current study although the configuration of the ion chamber are somewhat different. This study was done to assess the advantage of a hemispherical chamber.

\section{FISSION FRAGMENT STOPPING POWER}

For ranges which are long compared with the dimensions of the ion chamber, the pulse amplitude from either a fission fragment or an alpha particle depends primarily on its stopping power. By contrast when the ranges are short, the entire kinetic energy of the particle is deposited in the gas. The pulse amplitude is then primarily related to the energy of the particle. The ranges and initial stopping power for the two dominant alpha emissions from Cf-252 and a few of the fission products are shown in Table 1.

The spontaneous fission of Cf-252 produces many hundreds of different fission fragments. To predict which of these fission fragments would produce the smallest pulse a simple relation between the mass $m, Z$ number, energy of the fission product $E$ and pulse amplitude is desired. With respect to these parameters, the stopping power is roughly related as $\frac{d E}{d x} \propto \frac{m Z^{2}}{E}$.

In addition, the energy and mass are related. In general the fission-fragment kinetic energy decreases with mass. The minimum pulse should therefore result from the lightest fission fragment with the lowest $Z$ number. Although exceedingly rare, $\mathrm{Cr}-66$ is the lightest fission fragment from $\mathrm{Cf}-252$ with the lowest $Z$ number. It should therefore produce the smallest pulse from fission products in the ion chamber. This fission fragment is shown in Table 1. The minimum pulse should occur when this fragment crosses the $1 \mathrm{~mm}$ gap in the ion chamber normal to the plates. The energy deposited will be $4 \mathrm{MeV}$ in this case. The most probable fission product with this mass, Co-66, is also shown for comparison.

For comparison, the most massive fragments have a mass of $172 \mathrm{amu}$. Holmium is the most probable of these. The fragments Rh-105 and Ba-140 are intended to represent the most probable of the light and heavy fission fragments in Table 1 shown for comparison.

Table 1. Range and initial stopping power for $\mathrm{Cf}-252$ alpha particles and some fission products in argon at $1 \mathrm{~atm}$ at room temperature

\begin{tabular}{|l|r|r|r|r|}
\hline & \multicolumn{1}{|c|}{$\begin{array}{c}\mathrm{A} \\
(\mathrm{g} / \mathrm{mol})\end{array}$} & $\begin{array}{c}\mathrm{E} \\
(\mathrm{MeV})\end{array}$ & $\begin{array}{c}\text { Range } \\
(\mathrm{mm})\end{array}$ & \multicolumn{1}{c|}{$\begin{array}{c}\mathrm{dE} / \mathrm{dx} \\
(\mathrm{MeV} / \mathrm{mm})\end{array}$} \\
\hline Alpha & 4 & 6.08 & 52.5 & $7.74 \mathrm{E}-02$ \\
Alpha & 4 & 6.12 & 53.0 & $7.71 \mathrm{E}-02$ \\
Cr-66 & 66 & 108 & 35.0 & 3.91 \\
Co-66 & 66 & 108 & 34.6 & 4.47 \\
Rh-105 & 105 & 105 & 23.1 & 6.62 \\
Ba-140 & 140 & 84 & 19.9 & 6.65 \\
Ho-172 & 172 & 49 & 15.2 & 6.45 \\
\hline
\end{tabular}


The stopping power depends on the density of the stopping medium. In the Cf-252 ionchamber, the stopping medium is a mixture of $97 \%$ argon and $3 \% \mathrm{CO}_{2}$. The $\mathrm{CO}_{2}$ is ignored for this calculation. The density of argon was estimated from

$$
\rho=A \frac{n}{V}=A \frac{P}{R T}=1.654 \times 10^{-3} \mathrm{~g} / \mathrm{cm}^{3}
$$

where

$$
\begin{aligned}
& A=39.948 \mathrm{~g} / \mathrm{mol} \\
& P=1 \mathrm{~atm} \\
& T=70 F=294.26 \mathrm{~K} .
\end{aligned}
$$

A typical data book (Ref. 6) value for the density of argon adjusted from STP (1 atm, $273.15 \mathrm{~K})$ is

$$
1.7837 \mathrm{~g} / \mathrm{l} \frac{273.15 K}{294.26 K}=1.6557 \mathrm{~g} / \mathrm{l} \text {. }
$$

\section{ALPHA STOPPING POWER}

The discrimination of alpha pulses depends on the ratio of the amplitude of fission product pulses to alpha pulses. Figure 2 shows the energy lost by an alpha over relevant distances traveled in argon as well as the stopping power at these distances. Although the stopping power is not constant, it only changes from $0.08 \mathrm{MeV} / \mathrm{mm}$ to about 0.09 $\mathrm{MeV} / \mathrm{mm}$ over relevant distances.

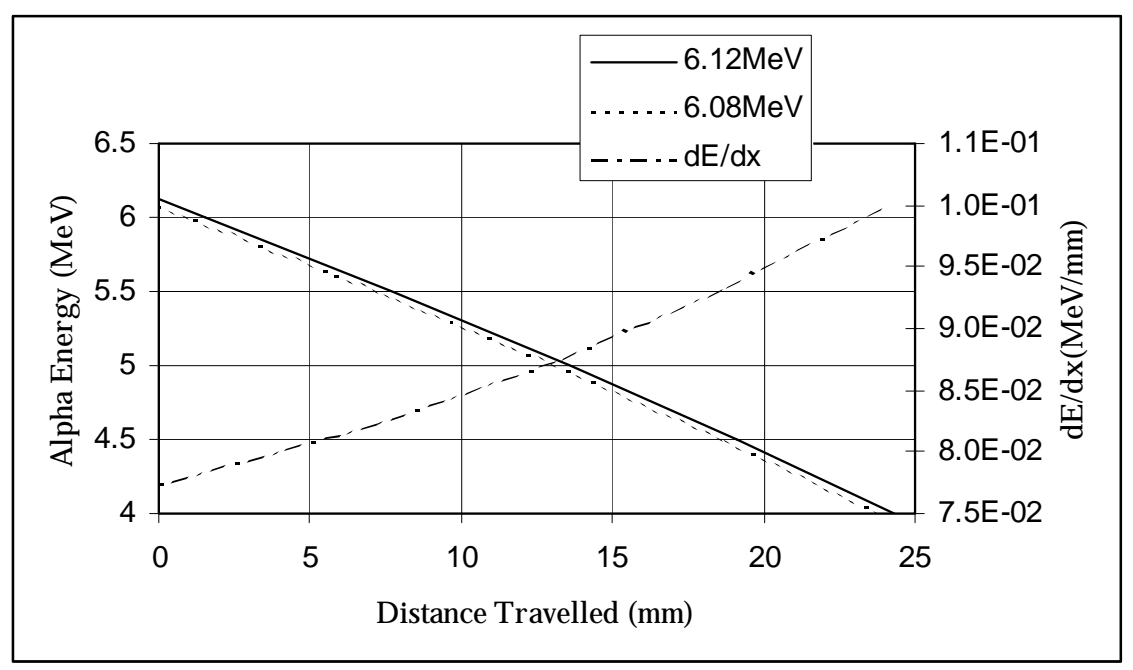

Figure 2. The energy of an alpha particle as it traverses argon at 1 atm at room temperature. The stopping power, which is the slope of this curve, is also shown. 


\section{MAXIMUM PATH LENGTH}

The maximum alpha pulse results when the alpha is emitted in the direction of the longest path length $r$ in the ion chamber as shown in Figure 1. Let the gap between the detector electrodes in either configuration be represented as $d$. The proposed value in both cases is $1 \mathrm{~mm}$. Let $R$ represent the radius of the cylinder in the parallel-plate configuration or the radius of the outer hemisphere in the two-concentric-hemisphere configuration. A nominal value for $R$ is $12.5 \mathrm{~mm}$. The maximum path length is then

$$
\begin{aligned}
& r=\sqrt{2 R d-d^{2}} \approx 4.9 \mathrm{~mm} \text { for a hemispherical detector. } \\
& r=\sqrt{(R+0.5 \mathrm{~cm})^{2}+d^{2}} \approx R+0.5 \mathrm{~cm} \approx 17.5 \mathrm{~cm} \text { for a parallel-plate detector. }
\end{aligned}
$$

Figure 3 shows the ratio of the maximum alpha pulse to the minimum fission pulse as the radius $R$ is varied. The gap $d$ is held constant at $1 \mathrm{~mm}$. The ratio improves as $R$ is decreased. However, a minimum $R$ of $6 \mathrm{~mm}$ is necessary to accommodate the Cf-252 spot and enough distance to collect charge from fission at the edge of the spot.

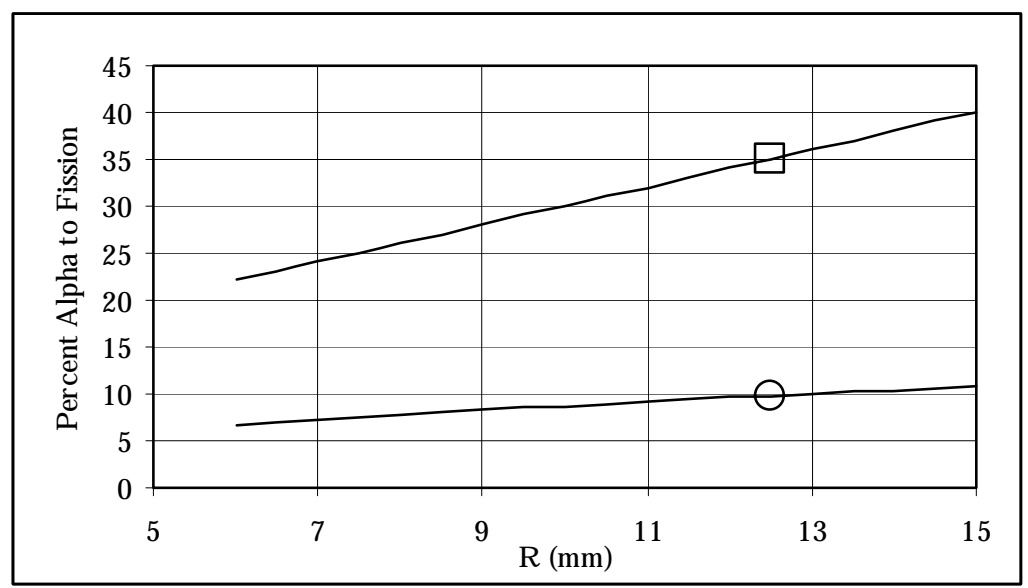

Figure 3. Percent minimum fission pulse to maximum alpha pulse in a parallel-plate and hemispherical fission chamber with a gap of $1 \mathrm{~mm}$ and varying $R$. Data for the $12.5 \mathrm{~mm}$ radius are shown as a square and circle respectively.

\section{RATIO OF PULSE AMPLITUDES}

Because the particle ranges are large compared to the dimensions of the ion chamber, the minimum fission pulse can be estimated as $3.9 \mathrm{MeV} / \mathrm{mm}$ times the gap distance. The maximum single alpha pulse can similarly be estimated as $0.085 \mathrm{MeV} / \mathrm{mm}$ times the maximum path length in the ion chamber. In either configuration the gap is $1 \mathrm{~mm}$. The minimum fission pulse is therefore $4 \mathrm{MeV}$ in both cases. 
Hemispherical case:

In the hemispherical ion chamber, an alpha will deposit $0.4 \mathrm{MeV}$ of energy as it traverses the maximum distance. This is $10 \%$ that of the minimum pulse from a fission fragment. This data point is shown in Figure 3 as a circle for a $12.5 \mathrm{~mm}$ radius.

Parallel-Plate Case:

An alpha will deposit from 1.3 to $1.4 \mathrm{MeV}$ traversing the maximum distance in the parallel-plate ion chamber. This is $35 \%$ that of the minimum pulse from a fission fragment. This data point is shown in Figure 3 as a square for a $12.5 \mathrm{~mm}$ radius.

\section{CONCLUSION}

The ratio of minimum fission pulse to the maximum alpha pulse is a factor of four larger for the hemispherical chamber compared to the parallel-plate chamber. It should therefore be easier to discriminate against alpha pulses in the hemispherical chamber.

\section{REFERENCES}

1. J. T. Mihalczo, "Use of $252 \mathrm{Cf}$ as a Randomly Pulsed Neutron Source for Prompt Neutron Decay Measurements," Doctoral Dissertation, University of Tennessee, Knoxville, 1970.

2. N. W. Hill, J. T. Mihalczo, J. W. Allen, M. M. Chiles, "Optimization of Nanosecond Fission Ion Chambers for Reactor Physics Applications," IEEE Nuclear Science, vol. NS-22, 686 (February 1975).

3. M. M. Chiles, J. T. Mihalczo, C. E. Fowler, "Small, Annular, Double-Contained ${ }^{252} \mathrm{Cf}$ Fission Chamber for Source-Driven Subcriticality Measurements," IEEE Nuclear Science, 40, 816 (August 1993).

4. Ziegler, J. F., Biersack, J. P., Littmark, U., The Stopping and Range of Ions in Solids, Pergamon Press, NY 1985, 1996.

5. Ziegler, J. F., SRIM 2000.39.

6. Lide, D. R., "CRC Handbook of Chemistry and Physics, $78^{\text {th }}$," CRC Press, Boca Raton 1998. 


\section{DISTRIBUTION}

Polytechnic of Milan

S. A. Pozzi

UT-Battelle

L. G. Chiang

A. C. Gehl

J. K. Mattingly

J. T. Mihalczo

J. A. Mullens

J. A. Ramsey

J. D. White

M. C. Wright 